\title{
What we need to know about antidepressants in unipolar major depression
}

\author{
Sarah Barber, Andrea Cipriani \\ Department of Psychiatry, University of Oxford, Oxford, UK
}

Miss Sarah Barber is a medical student at the University of Oxford. Professor Andrea Cipriani is Associate Professor at the Department of Psychiatry, University of Oxford.

\section{Correspondence to:}

Andrea Cipriani

Department of Psychiatry, University of Oxford

Warneford Hospital

Oxford, UK

Tel: +44 (0)1865 226393

Email: andrea.cipriani@psych.ox.ac.uk 


\section{Background}

Estimating the prevalence of depression is a challenge. Retrospective studies suggest a lifetime risk of around $10 \%$, but prospective data indicates it could be as high as $30 \%$ (Andrews et al., 2005). Even if psychotherapies have shown to be effective, the mainstay of treatment is antidepressants. A recent study found that $9 \%$ of British adults had taken antidepressants in the last year (Lewer et al., 2015), in keeping with a sharp increase in prescriptions in the last decade (Gusmão et al., 2013). It is of vital importance that hospital doctors understand the evidence base for pharmacological interventions in depression, as they increasingly encounter patients who have taken or are taking antidepressants. Moreover, depression is associated with longer lengths of stay in hospital, and poorer outcomes (Prina et al., 2012). Hospital doctors must meet the challenge of caring for their patients' mental, as well as physical health. This editorial is aimed at presenting the best available evidence to answer four questions of clinical relevance with respect to unipolar, non-treatment resistant, acute major depression: do antidepressants work? Who should be treated? Which is, if any, the best antidepressant to prescribe? How long antidepressants should be continued?

\section{Do antidepressants work?}

Regulatory agencies, like the US Food and Drug Administration or the European Medicine Agency, license drugs which demonstrate superior efficacy to placebo in at least two placebo-controlled randomized studies. This is true for all pharmacological interventions in medicine and in the past 30 years many drugs have been approved for the treatment of major depression. Notwithstanding this and despite the rise in use of antidepressants over the past decades, there is still a long lasting debate in psychiatry as to whether antidepressants have any real clinical benefit over placebo (Cipriani \& Geddes, 2014). On average, 50 to $60 \%$ of adults in trials respond to antidepressants and about 30 to $40 \%$ respond to placebo, however the difference between drug and placebo decreases when unpublished 
data are included (Barbui et al., 2008). Some even argue that unblinding due to adverse effects leads to a increased effect in the drug group, over-estimating the efficacy of antidepressants (Moncrieff et al., 2004). If this were the case, one would expect that trials showing larger differences in adverse events between drug and placebo would show larger differences in efficacy; interestingly, a meta-analysis by Barth et al. (2016) failed to demonstrate this. Results from this study do not support the hypothesis that efficacy of antidepressants over placebo in randomised trials is biased by unblinding due to adverse effects. These findings were consistent across different efficacy outcomes and statistical methods. However, due to fundamental methodological challenges inherent to placebo-controlled study design, these results should be interpreted with caution and considered only as preliminary evidence.

\section{Who should be treated?}

Antidepressants work "on average" for people with unipolar major depression, which means that they may be effective for some patients but not others. There are clinical moderators of response to treatment. For example a meta-analysis of individual patient data from six studies showed that the benefit of medication increases with the baseline severity of illness (Fournier et al., 2010). NICE (2009) does not recommend the routine use of antidepressants for the treatment of subthreshold depressive symptoms or major depression of mild severity (see Table 1 for definitions) on the basis of the results from a meta-analysis, which included only patients with so-called minor depression and found no significant difference in efficacy or acceptability between placebo and antidepressants (Barbui et al., 2011). Despite some limitations, such as the short follow up duration and incomplete outcome assessment in the included studies, these results make it difficult to justify the prescription of antidepressants, with all their side effects, to patients with milder illness.

\section{Which antidepressant should be used?}


It is easy to think of antidepressants as classes of drugs, which have similar efficacy and acceptability. Indeed, this is the position adopted by current guidelines, which concludes there is "little difference in efficacy between individual drugs" (NICE, 2009; Cleare et al., 2015; Malhi et al., 2015). This view is supported by a meta-analysis published by Gartlehner et al in the Annals of Internal Medicine (Gartlehner et al., 2011), but is in contradiction to a network meta-analysis published in the Lancet in 2009 (Cipriani et al., 2009). Both these analyses used a multiple-treatments meta-analysis technique, allowing for direct and indirect comparisons between antidepressants included in the systematic review (namely selective serotonin reuptake inhibitors (SSRIs) and serotonin-noradrenaline reuptake inhibitors). Furthermore, both found statistically significant differences between treatments. Yet, the conclusions widely differed - Gartlehner et al did not support a recommendation on the basis of efficacy, in keeping with NICE, yet Cipriani et al find in favour of escitalopram and sertraline.

The clinical interpretation of study findings may vary and it is worth remembering that the practice of evidence-based medicine implies the integration of the best available research data with individual clinical expertise and patients' preferences and values. Evidence-based practice is not "cookbook" medicine and, similarly, ranking antidepressants is not aimed at providing clinicians and patients with a list of drugs to mechanically scroll down from top to bottom. Results from the Lancet 2009 review challenged the standard thinking that most antidepressants are of similar average efficacy and tolerability (Cipriani et al., 2009) and, even when significant differences were observed between drugs, these tended to be minimized and considered clinically insignificant (Gartlehner et al., 2011). We think this is a comfortable position for industry as it sets a low threshold for the introduction of new agents which can then be marketed on the basis of small differences in specific adverse effects rather than on clear advantages in terms of overall average efficacy and acceptability (Geddes \& Cipriani, 2015). Nonetheless, accumulating evidence suggests that antidepressants may vary not only in their efficacy and acceptability, but also in terms of hard outcome measures, for example the risk of suicide (Stone et 
al., 2009). Efficacy is not the sole consideration when choosing a drug, tolerability must always be taken into account. NICE recommend SSRIs as first line on the basis of their superior side-effect profile to other antidepressants. In particular, SSRIs are safer in overdose and along with the other second-generation antidepressants are associated with less drowsiness and anticholinergic side effects than tricyclics and mono-amine oxidase inhibitors. Is there any evidence of differences in tolerability between secondgeneration antidepressants? Future studies should specifically address this issue using a comparative statistical technique (like a network meta-analysis), however this is difficult to do because adverse events are not collected systematically in randomized controlled trials, with a high risk of selective reporting bias.

\section{How long should antidepressants be continued?}

Over half of people with major depression will have more than one episode, with each further episode increasing the risk of relapse (NICE, 2009). However, continuing antidepressant treatment over six months to three years can reduce the risk of relapse up to more than $50 \%$ (Reid \& Barbui, 2010). As always, potential limitations should be taken into account when interpreting these results. Most of the 31 studies included in the analysis were of a discontinuation design, whereby responders are randomized to either continuing treatment or receiving placebo. Some researchers argued that switching to placebo increases the risk of withdrawal symptoms that mimic a relapse, thereby overestimating the benefit of medication. However, a meta-analysis of six trials of SSRIs vs placebo, with no discontinuation design but longer follow up, demonstrated an advantage of active medication over placebo at 6 months (Deshauer et al., 2008). Current NICE guidelines recommends continuing treatment for a minimum of 6 months, but 2 years if there is a high risk of relapse. Of course, it is the patient who, with their psychiatrist or GP, will ultimately decide if the benefit outweighs the potential harms of side effects. 


\section{Conclusions}

This editorial has considered four key "pragmatic" questions, which are at the centre of the debate in the scientific literature about antidepressants in the treatment of unipolar, non-resistant major depression. From a clinical point of view, it is clear that these questions cannot be considered in isolation. For example, we cannot answer the question "do antidepressants work?", without knowing for who, for how long, and - potentially most importantly - which drug? Whilst relevant meta-analyses consistently found statistically significant differences between drugs in terms of efficacy and acceptability, there is remains no agreement as to what constitutes a clinically significant effect for the patient (Cleare et al., 2015). Methodological development of evidence synthesis can probably help overcome this uncertainty (Cipriani et al., 2013). Future network meta-analyses should incorporate as many drugs as possible (old and new antidepressants, together with placebo), in order to better inform clinical practice, and not only in developed countries. 


\section{References}

Andrews G, Poulton R, Skoog, I (2005) Lifetime risk of depression: restricted to a minority or waiting for most? Br J Psychiatry 187(6): 495-6.

Barbui C, Cipriani A, Patel V, Ayuso-Mateos JL, van Ommeren M (2011) Efficacy of antidepressants and benzodiazepines in minor depression: systematic review and meta-analysis. Br J Psychiatry 198(1): $11-6$.

Barbui C, Furukawa TA, Cipriani A (2008) Effectiveness of paroxetine in the treatment of acute major depression in adults: a systematic re-examination of published and unpublished data from randomized trials. CMAJ 178(3): 296-305.

Barth M, Kriston L, Klostermann S, Barbui C, Cipriani A, Linde K (2016) Efficacy of selective serotonin reuptake inhibitors and adverse events: meta-regression and mediation analysis of placebocontrolled trials. Br J Psychiatry 208(2): 114-9.

Cipriani A, Furukawa TA, Salanti G, et al (2009) Comparative efficacy and acceptability of 12 newgeneration antidepressants: a multiple-treatments meta-analysis. Lancet 373(9665): 746-58.

Cipriani A, Geddes JR (2014) Placebo for depression: we need to improve the quality of scientific information but also reject too simplistic approaches or ideological nihilism. BMC Medicine 12: 105.

Cipriani A, Higgins JPT, Geddes JR, Salanti G (2013) Conceptual and technical challenges in network meta-analysis. Ann Intern Med, 159(2): 130-7.

Cleare A, Pariante CM, Young AH, et al (2015) Evidence-based guidelines for treating depressive disorders with antidepressants: A revision of the 2008 British Association for Psychopharmacology guidelines. J Psychopharmacol 29(5): 459-525.

Deshauer D, Moher D, Fergusson D, Moher E, Sampson M, Grimshaw J (2008) Selective serotonin reuptake inhibitors for unipolar depression: a systematic review of classic long-term randomized controlled trials. CMAJ 178(10): 1293-301.

Fournier JC, DeRubeis RJ, Hollon SD, et al (2010) Antidepressant drug effects and depression severity: a patient-level meta-analysis. JAMA 303(1): 47-53.

Gartlehner G, Hansen RA, Morgan LC, et al (2011) Comparative benefits and harms of second-generation antidepressants for treating major depressive disorder: an updated meta-analysis. Ann Intern Med 155(11): 772-85.

Geddes JR, Cipriani A (2015) Time to abandon placebo control in pivotal phase III trials? World Psychiatry 14(3): 306-7.

Gusmão R, Quintão S, McDaid D, et al (2013) Antidepressant Utilization and Suicide in Europe: An Ecological Multi-National Study. PloS One 8(6): e66455. 
Lewer D, O'Reilly C, Mojtabai R, Evans-Lacko S (2015) Antidepressant use in 27 European countries: associations with sociodemographic, cultural and economic factors. Br J Psychiatry 207(3): 221-6.

Malhi GS, Bassett D, Boyce P, et al (2015) Royal Australian and New Zealand College of Psychiatrists clinical practice guidelines for mood disorders. Aust N Z J Psychiatry 49(12): 1087-206.

Moncrieff J, Wessely S, Hardy R (2004) Active placebos versus antidepressants for depression. Cochrane Database Syst Rev 1: CD003012.

National Institute for Health and Clinical Excellence. Depression: the treatment and management of depression in adults (update) (2009) Clinical guideline 90. http://guidance.nice.org.uk/CG90

Prina AM, Deeg D, Brayne C, Beekman A, Huisman M (2012) The association between depressive symptoms and non-psychiatric hospitalisation in older adults. PloS One 7(4): e34821.

Reid S, Barbui C (2010) Long term treatment of depression with selective serotonin reuptake inhibitors and newer antidepressants. BMJ 340: c1468.

Stone, M, Laughren T, Jones ML, et al (2009) Risk of suicidality in clinical trials of antidepressants in adults: analysis of proprietary data submitted to US Food and Drug Administration. BMJ 339: b2880. 
Table 1: Depression definitions used in the NICE guidelines (NICE 2009) based on DSM-IV criteria*

\begin{tabular}{|l|l|}
\hline Category & Definition \\
\hline or “minor" depression & Fewer than 5 symptoms of depression \\
\hline Mild depression & Few, if any, symptoms in excess of the 5 required to make the \\
& impairment \\
\hline Moderate depression & $\begin{array}{l}\text { Symptoms or functional impairment are between 'mild' and } \\
\text { 'severe' }\end{array}$ \\
\hline Severe depression & Most symptoms, and the symptoms markedly interfere with \\
& functioning. Can occur with or without psychotic symptoms \\
\hline
\end{tabular}

Legend: *NICE guidelines (NICE 2009) are based on DSM-IV criteria, where the distinction between mild, moderate and severe depression depends on the degree functional impairment, as shown above. In the ICD-10, however, the criteria depend on the number of symptoms, with a lower threshold for mild depression (4 symptoms), and 5-6 and 7 or more symptoms required for moderate and severe depression respectively. 